\title{
Instability of cosmological event horizons of non-static global cosmic strings
}

\author{
Anzhong Wang * \\ Departamento de Física Teórica, Universidade do Estado do Rio de Janeiro, \\ Rua São Francisco Xavier 524, Maracanã, 20550-013 Rio de Janeiro - RJ, Brazil \\ and \\ Observatório Nacional - CNPq, Rua General José Cristino 77, \\ São Cristóvão, 20921-400 Rio de Janeiro - RJ, Brazil \\ José A.C. Nogales ${ }^{\dagger}$ \\ Instituto de Física, Universidade Federal Fluminense, \\ Av. Litorânea s/n - Boa Viagen, CEP 24210-340 Niterói - RJ, Brazil \\ and \\ Instituto de Física, Universidad Mayor de San Andres (UMSA), \\ Casilla 3553, La Paz - Bolivia
}

*e-mail address: wang@symbcomp.uerj.br

${ }^{\dagger}$ e-mail:jnogales@portela.if.uff.br 


\begin{abstract}
The stability of the cosmological event horizons found recently by Gregory [Phys. Rev. D54, 4955 (1996)] for a class of non-static global cosmic strings is studied. It is shown that they are not stable to both test particles and physical perturbations. In particular, the back reaction of the perturbations of null dust fluids will turn them into spacetime singularities. The resulted singularities are strong in the sense that the distortion of test particles diverges logarithmically when these singular hypersurfaces are approaching.
\end{abstract}

PACS numbers: 98.80.Cq, 04.20Jb, 04.40.+c. 


\section{INTRODUCTION}

Topological defects formed in the early Universe have been studied exclusively [[1], since the pioneering work of Kibble [2]. They were formed during phase transitions of the Universe, where the degenerated vacua acquired non-zero expectation values. Depending on the topology of the vacua, the defects could be domain walls, cosmic strings, monopoles, textures, or the hybrids of them. Among these defects, cosmic strings have received particular attention mainly because of their cosmological implications: They might provide the seeds for the formations of galaxies and the large-scale structure of the Universe [1].

Cosmic strings are further classified as local (gauge) and global strings, according to whether they arise from a local symmetry breaking or a global symmetry breaking. These two kinds of strings have very different properties. In particular, the spacetime of a local (static) string is well behaved and asymptotically approaches a conical spacetime [3], while the spacetime of a global static string is necessarily singular at a finite distance from the symmetric axis, and its deficit angle diverges logarithmically [4]. It is this undesirable property that make global strings very difficult to use, and most studies of cosmic strings have been restricted only to local strings [1].

However, local strings are tightly constrained by their contribution to the gravitational radiation background [5, 6], while global cosmic strings cir- 
cumvent this constraint and may have similar cosmological implications [0] Lately, Banerjee et al. [8] and Gregory [9] studied non-static global strings, and some interested results were found. In particular, Gregory showed that the spacetime singularities usually appearing in the static case can be replaced by cosmological event horizons $(\mathrm{CEH})$ t. This result is very important, as it may make the structure formation scenario of cosmic strings more likely, and may open a new avenue to the study of global strings.

In this paper, we shall study the stability of the CEHs found above by Gregory [9], and shall show that in general they are not stable against small perturbations, instead are turned into spacetime singularities. Does this mean that the hope that time-dependence might remove the singular nature of global static string spacetimes is already found negative? We think that it is not, at least as far as the work of Gregory is concerned. As a matter of fact, Gregory considered a very particular case: the energy-momentum tensor of the string is still time-independent. As a result, no gravitational and particle radiation exists. For more general case, one would expect that CEHs may not be formed at all, or even they are formed but stable. As we know, in the cylindrical case gravitational and particle radiation in general always exists. It is plausible to expect that in some situations the radiation

\footnotetext{
${ }^{1}$ Note that Gregory called the horizons as event horizons. However, to be distinguishable with the ones of black holes, following Gibbons and Hawking [10], we call them cosmological event horizons.
} 
is so strong that the gravitational field is well dilated before any spacetime singularity or horizon is formed.

The rest of the paper is organized as follows: In Section II we shall briefly review the main properties of the spacetimes studied by Gregory [9], while in Section III null dust fluids of test particles are studied, which indicate some singularity behavior of the spacetimes near the CEHs. In Section IV we consider "physical" perturbations of real particles, and confirm the results obtained in Section III. Here "physical" is in the sense that the back reaction of the perturbations are taken into account. Finally in Section V we derive our main conclusions.

\section{SPACETIME FOR NON-STATIC GLOBAL COSMIC STRINGS}

For a straight cosmic string, we can always choose a coordinate system that is comoving with the string so that the spacetime in this system has a cylindrical symmetry. If additionally we require that the string has no rotation, the metric for such a spacetime takes the general form [11]

$$
d s^{2}=e^{2(\gamma-\psi)}\left(d t^{2}-d R^{2}\right)-e^{2 \psi} d z^{2}-\alpha^{2} e^{-2 \psi} d \theta^{2},
$$

where $\gamma, \psi$ and $\alpha$ are functions of $t$ and $R$ only, and $t, R, z$ and $\theta$ are the usual cylindrical coordinates. 
By requiring that the string has fixed proper width and that the spacetime has boost symmetry in the $(t, z)$-plane, Gregory managed to show that the spacetime for a $\mathrm{U}(1)$ global string (vortex) is given by [9]

$$
\gamma=2 a(R)+b(t), \quad \psi=a(R)+b(t), \quad \alpha=c(R) e^{a(R)+b(t)},
$$

where $a(R)$ and $c(R)$ are two arbitrary functions, and $b(t)$ is given by

$$
b(t)= \begin{cases}\ln [\cosh (\beta t)], \pm \beta t, & b_{0}>0 \\ b_{1} \ln t, & b_{0}=0 \\ \ln [\cos (\beta t)], & b_{0}<0\end{cases}
$$

where $b_{0}$ and $b_{1}$ are arbitrary constants, and $\beta \equiv \sqrt{\left|b_{0}\right|}$. from Eqs.(2.1) and (2.2) we can see that, by introducing a new radial coordinate $r$ via the relation

$$
r=\int^{r} e^{a(R)} d R
$$

the metric (2.1) can be written in the form

$$
d s^{2}=e^{2 A(r)} d t^{2}-d r^{2}-e^{2[A(r)+b(t)]} d z^{2}-C^{2}(r) d \theta^{2},
$$

where $A(r) \equiv a(R(r))$ and $C(r) \equiv c(R(r))$. This is exactly the form used by Gregory [9].

As shown by Gregory, the spacetime inside the core of a string is always singular at a finite distance for the cases $b_{0} \leq 0$, and has a $\mathrm{CEH}$ for $b_{0}>0$. In fact, in the latter case the metric coefficients have the asymptotic behavior

$$
e^{A(r)} \sim \beta\left(r_{0}-r\right), \quad C(r) \sim C_{0}+O\left(r_{0}-r\right)^{2},
$$


as $r \rightarrow r_{0}^{-}$, where $C_{0}$ is a constant [cf. Eq.(3.14) in Ref. 9]. For the choice $b(t)=\ln [\cosh (\beta t)]$, the corresponding metric takes the form

$$
d s^{2}=\beta^{2}\left(r_{0}-r\right)^{2}\left[d t^{2}-\cosh ^{2}(\beta t) d z^{2}\right]-d r^{2}-C_{0}^{2} d \theta^{2}
$$

in the neighborhood of the the hypersurface $r=r_{0}$. One can show that the singularity appearing at $r=r_{0}$ in Eq. (2.7) is a coordinate one. This can be seen, for example, by making the following coordinate transformations

$$
\begin{aligned}
X & =\left(r_{0}-r\right) \cosh (\beta t) \cos (\beta z) \\
Y & =\left(r_{0}-r\right) \cosh (\beta t) \sin (\beta z), \\
T & =\left(r_{0}-r\right) \sinh (\beta t),
\end{aligned}
$$

then the metric (2.7) is brought to the form

$$
d s^{2}=d T^{2}-d X^{2}-d Y^{2}-C_{0}^{2} d \theta^{2}
$$

which is locally Minkowski. Thus, the singularity at $r=r_{0}$ in the coordinates $\{t, r, z, \theta\}$ is indeed a coordinate singularity and represent a cone-like $\mathrm{CEH}$ in the coordinates $\{T, X, Y, \theta\}$, as one can see from Eq.(2.8) that the hypersurface $r=r_{0}$ is mapped to

$$
X^{2}+Y^{2}=T^{2},\left(r=r_{0}\right)
$$

For the details, we refer readers to [9]. 
For $b(t)= \pm \beta t$, the corresponding metric takes the form

$$
d s^{2}=\beta^{2}\left(r_{0}-r\right)^{2}\left[d t^{2}-e^{ \pm \beta t} d z^{2}\right]-d r^{2}-C_{0}^{2} d \theta^{2}
$$

One can show that the singularities appearing at $r=r_{0}$ in the above metric also represent CEHs. In fact, if we make the coordinate transformations

$$
\begin{aligned}
T & =\frac{1}{2}\left(r_{0}-r\right)\left[\beta^{2} z^{2} e^{\beta t}+2 \sinh (\beta t)\right], \\
X & =\frac{1}{2}\left(r_{0}-r\right)\left[\beta^{2} z^{2} e^{\beta t}-2 \cosh (\beta t)\right], \\
Y & =\beta\left(r_{0}-r\right) z e^{\beta t},
\end{aligned}
$$

for $b(t)=+\beta t$, the metric (2.11) will be brought to the exact form of Eq.(2.9), while if we make the same transformations as those of Eq.(2.12) but with $t$ being replaced by $-t$ for $b(t)=-\beta t$, the metric (2.11) will be also brought to the same form, Eq.(2.9). Therefore, in the latter two cases the hypersurface $r=r_{0}$ all represents a $\mathrm{CEH}$. The topology of it is also conical in the Minkowiski-like coordinates $(T, X, Y, \theta)$, as one can show from Eq.(2.12) that Eq.(2.10) is satisfied, too.

Before proceeding further, we would like to note the following: a) For the coordinate transformations given by Eq.(2.8), the mapping between $(t, r, z)$ and $(T, X, Y)$ is not one-to-one, while the one given by Eq.(2.12) is. b) The nature of the CEHs in all these three cases is quite similar to that of the extreme Reissner-Nordström black hole [12], in the sense that across $r=r_{0}$ 
the coordinate $t$ remains time-like, while $r$ remains space-like. c) in the neighborhood $r=r_{0}$ but with $r>r_{0}$, Eq.(2.6) should be replaced by

$$
e^{A(r)} \sim \beta\left(r-r_{0}\right), \quad C(r) \sim C_{0}+O\left(r-r_{0}\right)^{2},\left(r>r_{0}\right)
$$

Substituting Eqs.(2.6) and (2.13) into Eq.(2.4), we find

$$
R= \begin{cases}\frac{1}{\beta} \ln \left[\beta\left(r-r_{0}\right)\right], & r>r_{0}, \\ -\frac{1}{\beta} \ln \left[\beta\left(r_{0}-r\right)\right], & r<r_{0},\end{cases}
$$

which shows that $R$ is a monotonically increasing function of $r$, except for the point $r=r_{0}$, at which $R$ diverges.

In the following, we shall consider the stability of these CEHs in two steps: First, in the next section we shall consider test particles near the CEHs along a line suggested by Helliwell and Konkowski (HK) in the study of the stability of quasiregular singularities [13], at the aim of generalizing the HK conjecture to the case of CEHs. Second, in Sec. IV we shall consider perturbations of null dust fluids. These perturbations are different from the ones studied in Sec. III, in the sense that the back reaction of them to the spacetime backgrounds will be taken into account.

\section{TEST NULL DUST FIELDS NEAR THE CEHs}

In a series of papers [13], HK studied the stability of quasiregular singularities by using test fields. In particular, they conjectured that if one introduces a 
test field whose energy-momentum tensor (EMT) calculated in a freely-falling frame mimics the behavior of the Riemann tensor components that indicate a particular type of singularity (quasiregular, non-scalar curvature, or scalar curvature), then a complete non-linear back-reaction calculation would show that this type of singularity actually occurs. Recently, this conjecture was further generalized to the stability of Cauchy horizons [14]. Clearly, if this conjecture is true, the stability analysis of spacetime singularities would be considerably simplified. In this section, using HK's ideas we shall study test null dust fields near the CEHs.

For a null dust fluid moving along the outgoing null geodesics defined by $n^{\mu}$ in the region $r \leq r_{0}$, the EMT takes the form

$$
T_{\mu \nu}^{-o u t}=\rho_{-}^{o u t} n_{\mu} n_{\nu}
$$

where $n_{\mu}$ is a null vector defined as that in Eq.(A.12). Then, from the conservation equations $T_{\mu \nu ; \lambda}^{-o u t} g^{\nu \lambda}=0$, we find

$$
e^{A}\left(\frac{\rho_{-}^{\text {out }}, r}{\rho_{-}^{\text {out }}}+3 A^{\prime}(r)\right)+\left(\frac{\rho_{-}^{\text {out }}, t}{\rho_{-}^{\text {out }}}+b^{\prime}(t)\right)=0,
$$

which has the solution

$$
\rho_{-}^{\text {out }}=\frac{\rho_{-}^{\text {out }(0)} e^{a_{1} t-b(t)}}{\left(r_{0}-r\right)^{3-a_{1} / \beta}},
$$

where ()$_{x}=\partial / \partial x$, a prime denotes the ordinary derivative with respect to the indicated argument, and $a_{1}$ and $\rho_{-}^{\text {out }(0)}$ are two constants, while $A(r)$ and 
$b(t)$ are given, respectively, by Eqs.(2.6) and (2.3). Projecting $T_{\mu \nu}^{-o u t}$ onto the PPON frame defined by Eqs.(A.6) and (A.7), we find that the non-vanishing components are given by

$$
\begin{aligned}
T_{(0)(0)}^{- \text {out }} & =T_{(1)(1)}^{- \text {out }}=T_{(0)(1)}^{- \text {out }}=\frac{\rho_{-}^{\text {out }(0)} e^{a_{1} t-b(t)}}{\left(r_{0}-r\right)^{3-a_{1} / \beta}} \\
& \times\left\{\frac{E^{2}}{\beta^{2}\left(r_{0}-r\right)^{2}}-\frac{E\left[E^{2}-\beta^{2}\left(r_{0}-r\right)^{2}\right]^{1 / 2}}{\left[\beta\left(r_{0}-r\right)\right]^{2}}-\frac{1}{2}\right\} .
\end{aligned}
$$

Clearly, for $b(t)=\ln [\cosh \beta t], \beta t$, we have to choose $a_{1}=\beta$ in order to have the perturbations be finite initially $(t=-\infty)$, while for $b(t)=-\beta t$, we have to choose $a_{1}=-\beta$, namely,

$$
a_{1}= \begin{cases}\beta, & b(t)=\ln [\cosh \beta t], \beta t, \\ -\beta, & b(t)=-\beta t .\end{cases}
$$

Eqs.(3.4) and (3.5) show that all the components diverge as $r \rightarrow r_{0}^{-}$for all the three different choices of $b(t)$, which indicates that if we take the back-reaction of the null dust fluid into account, the CEHs appearing on the hypersurface $r=r_{0}$ in the solutions (2.7) and (2.11) will be turned into spacetime singularities, provided that the HK conjecture still holds here. Since all the corresponding fourteen scalars constructed from the Riemann tensor are zero in the present case, the resulted singularities would be expected to be non-scalar curvature singularities.

In addition to the out-going null dust fluid, if there also exists an in-going null fluid moving along the null geodesics defined by $l^{\mu}$, i.e.,

$$
T_{\mu \nu}^{-i n}=\rho_{-}^{i n} l_{\mu} l_{\nu},
$$


where $l_{\mu}$ is defined by Eq.(A.12), then, from the conservation equations $T_{\mu \nu ; \lambda}^{-i n} g^{\nu \lambda}=0$, one can show that $\rho_{-}^{i n}$ is give by

$$
\rho_{-}^{i n}=\frac{\rho_{-}^{i n(0)} e^{a_{0} t-b(t)}}{\left(r_{0}-r\right)^{3+a_{0} / \beta}},
$$

where $a_{0}$ and $\rho_{-}^{i n(0)}$ are other two integration constants. Then, the nonvanishing tetrad components of $T_{\mu \nu}^{-i n}$ are given by

$$
\begin{aligned}
T_{(0)(0)}^{-i n} & =T_{(1)(1)}^{-i n}=-T_{(0)(1)}^{-i n}=\frac{\rho_{-}^{i n(0)} e^{a_{0} t-b(t)}}{\left(r_{0}-r\right)^{3+a_{0} / \beta}} \\
& \times\left\{\frac{E^{2}}{\beta^{2}\left(r_{0}-r\right)^{2}}+\frac{E\left[E^{2}-\beta^{2}\left(r_{0}-r\right)^{2}\right]^{1 / 2}}{\left[\beta\left(r_{0}-r\right)\right]^{2}}-\frac{1}{2}\right\} .
\end{aligned}
$$

Similar to the out-going case, to have the perturbations be finite initially $(t=-\infty)$, we have to choose $a_{0}=a_{1}$, where $a_{1}$ is given by Eq.(3.5). Then, from Eq.(3.8) we can see that these components also diverge. Since now we have

$$
T^{-\mu \nu} T_{\mu \nu}^{-}=2 \rho_{-}^{\text {out }} \rho_{-}^{\text {in }}=2 \rho_{-}^{\text {out }(0)} \rho_{-}^{\text {in }(0)} \frac{e^{2\left[a_{0} t-b(t)\right]}}{\left(r_{0}-r\right)^{6}},
$$

which always diverges as $r \rightarrow r_{0}^{-}$, we can see that the resulted singularities should be scalar curvature ones when the two null dust fluids are all present, where

$$
T_{\mu \nu}^{-} \equiv T_{\mu \nu}^{-o u t}+T_{\mu \nu}^{-i n}
$$

Similarly, we can consider test null dust fields in the region $r \geq r_{0}$, and will obtain the same conclusions. Thus, the above considerations suggest that all the CEHs appearing in the solutions (2.7) and (2.11) are not stable against perturbations for all the three different choices of $b(t)$ with $b_{0}>0$. 


\section{PERTURBATIONS NEAR THE CEHs}

In this section, let us consider perturbations of null dust fluids near the CEHs.

For the sake of convenience, we shall work with the coordinates $t$ and $R$, in terms of which the metrics (2.7) and (2.11) can be cast in the form

$$
d s^{2}=e^{-\Omega_{(0)}}\left(d t^{2}-d R^{2}\right)-e^{-h_{(0)}}\left[e^{\Phi_{(0)}} d z^{2}+e^{-\Phi_{(0)}} d \theta^{2}\right]
$$

where

$$
\begin{aligned}
& \Omega_{(0)}=2 \beta R, \quad h_{(0)}=\beta R-b(t)-\ln C_{0}, \\
& \Phi_{(0)}=-\beta R+b(t)-\ln C_{0},
\end{aligned}
$$

for $r \leq r_{0}$, and

$$
\begin{aligned}
& \Omega_{(0)}=-2 \beta R, \quad h_{(0)}=-\left[\beta R+b(t)+\ln C_{0}\right], \\
& \Phi_{(0)}=\beta R+b(t)-\ln C_{0},
\end{aligned}
$$

for $r \geq r_{0}$, and the function $b(t)$ is given by Eq.(2.3).

As shown in [15], the null dust fluids given by

$$
T_{\mu \nu}=\rho^{i n} l_{\mu} l_{\nu}+\rho^{o u t} n_{\mu} n_{\nu},
$$

have contributions only to the metric coefficients $g_{t t}$ and $g_{R R}$. Specifically, if we set

$$
\{\Omega, h, \Phi\}=\left\{\Omega_{(0)}+f(u)+g(v), h_{(0)}, \Phi_{(0)}\right\}
$$


the metric

$$
d s^{2}=e^{-\Omega}\left(d t^{2}-d R^{2}\right)-e^{-h}\left(e^{\Phi} d z^{2}+e^{-\Phi} d \theta^{2}\right)
$$

will satisfy the Einstein field equations $R_{\mu \nu}-g_{\mu \nu} R / 2=T_{\mu \nu}$, with $\rho^{\text {out }}$ and $\rho^{\text {in }}$ being given, respectively, by

$$
\rho^{\text {out }}=g^{\prime}(v) h_{, v}, \quad \rho^{i n}=f^{\prime}(u) h_{, u}
$$

and now

$$
\begin{aligned}
l_{\mu} & =e^{-\Omega / 2}\left(\delta_{\mu}^{t}+\delta_{\mu}^{R}\right), \quad n_{\mu}=e^{-\Omega / 2}\left(\delta_{\mu}^{t}-\delta_{\mu}^{R}\right), \\
u & \equiv \frac{t+R}{\sqrt{2}}, \quad v \equiv \frac{t-R}{\sqrt{2}}
\end{aligned}
$$

and $f(u)$ and $g(v)$ are arbitrary functions of their indicated arguments. Note that although $T_{\mu \nu}$ now takes the same form as that considered in the last section, it has fundamental difference: now it acts as a source of the spacetime. As a result, the back reaction of it is automatically fully taken into account. When $f(u), g(v)$ and their first derivatives are very small, the two dust fluids can be considered as perturbations of the spacetime given by Eqs.(4.1) - (4.3). In the following, let us consider the three cases $b(t)=\ln [\cosh (\beta t)],+\beta t,-\beta t$, separately.
A. $b(t)=\ln [\cosh (\beta t)]$

In this case, Eqs.(4.2) - (4.5) yield

$$
\Omega=f^{-}(u)+g^{-}(v)+2 \beta R,
$$




$$
\begin{aligned}
h & =\beta R-\ln [\cosh (\beta t)]-\ln C_{0}, \\
\Phi & =-\beta R+\ln [\cosh (\beta t)]-\ln C_{0}
\end{aligned}
$$

for $r \leq r_{0}$, and

$$
\begin{aligned}
\Omega & =f^{+}(u)+g^{+}(v)-2 \beta R, \\
h & =-\left\{\beta R+\ln [\cosh (\beta t)]+\ln C_{0}\right\}, \\
\Phi & =\beta R+\ln [\cosh (\beta t)]-\ln C_{0},
\end{aligned}
$$

for $r \geq r_{0}$. Substituting Eqs.(4.9) and (4.10) into Eq.4.4), we find

$$
\begin{aligned}
& \rho_{-}^{\text {out }}=-\frac{\beta e^{\beta t} g^{-^{\prime}}(v)}{\sqrt{2} \cosh \beta t}, \quad \rho_{-}^{\text {in }}=\frac{\beta e^{-\beta t} f^{-^{\prime}}(u)}{\sqrt{2} \cosh \beta t},\left(r \leq r_{0}\right), \\
& \rho_{+}^{\text {out }}=\frac{\beta e^{-\beta t} g^{+^{\prime}}(v)}{\sqrt{2} \cosh \beta t}, \quad \rho_{+}^{\text {in }}=-\frac{\beta e^{\beta t} f^{+^{\prime}}(u)}{\sqrt{2} \cosh \beta t},\left(r \geq r_{0}\right) .
\end{aligned}
$$

Note that it is not necessary to take $f^{-}(u)$ and $g^{-}(v)$ the same forms as $f^{+}(u)$ and $g^{+}(v)$, since now we consider the perturbations in both sides of the hypersurface $r=r_{0}$ independently. However, to have physically reasonable perturbations, we require

$$
\begin{aligned}
& g^{-^{\prime}}(v)<0, \quad g^{+^{\prime}}(v)>0 \\
& f^{-^{\prime}}(u)>0, \quad{f^{+\prime}}^{\prime}(u)<0
\end{aligned}
$$

so that $\rho_{ \pm}^{\text {out }}$ and $\rho_{ \pm}^{\text {in }}$ are all no negative.

When $f^{ \pm}(u), g^{ \pm}(v)$ and their first derivatives are very small, the radial time-like geodesics given by Eq.(A.6) would be a very good approximation of 
the corresponding ones of the metric (4.6). Consequently, the tetrad frames given by Eqs.(A.6) and (A.7) would serve well as the corresponding PPON of Eq.(4.6). Projecting the EMT onto this frame, we find that the non-vanishing tetrad components of it are given by

$$
\begin{aligned}
& T_{(0)(0)}^{ \pm}=T_{(1)(1)}^{ \pm}=\frac{1}{2}\left\{D_{+}(A) \rho_{ \pm}^{\text {in }}+D_{-}(A) \rho_{ \pm}^{\text {out }}\right\} \\
& T_{(0)(1)}^{ \pm}=T_{(1)(0)}^{ \pm}=\frac{1}{2}\left\{D_{+}(A) \rho_{ \pm}^{\text {in }}-D_{-}(A) \rho_{ \pm}^{\text {out }}\right\}
\end{aligned}
$$

where $\rho_{ \pm}^{\text {in }}, \rho_{ \pm}^{\text {out }}$ are given by Eqs.(4.11), and

$$
D_{ \pm}(A)=\left[\frac{E}{e^{A}} \pm \epsilon\left(\frac{E^{2}}{e^{2 A}}-1\right)^{1 / 2}\right]^{2},
$$

with $A$ being given by Eqs.(2.6) and (2.13). Clearly, as $r \rightarrow r_{0}$, these components all diverge. Note that in writing the above expressions we have used the fact that $f^{ \pm}(u)$ and $g^{ \pm}(v)$ are very small to set $\exp \left\{f^{ \pm}(u)+g^{ \pm}(v)\right\}=1$. This will be also the case for other two cases to be considered below. Combining Eqs.(4.11) with Eqs.(4.13) and (4.14), we can see that the perturbations are finite at the initial $t=-\infty$, but all will focus into a spacetime singularity when they arrive at $r=r_{0}$. That is, the perturbations turn the CEHs into spacetime curvature singularities. The nature of the singularity is a scalar one. This can be seen, for example, from the Kretshmann scalar,

$$
\mathcal{R}=R_{\alpha \beta \gamma \delta} R^{\alpha \beta \gamma}
$$




$$
= \begin{cases}-\frac{4 \beta^{2} e^{2\left[2 \beta R+f^{-}(u)+g^{-}(v)\right]}}{\cosh ^{2} \beta t} f^{-^{\prime}}(u) g^{-\prime}(v), & r \leq r_{0}, \\ \frac{4 \beta^{2} e^{-2\left[2 \beta R-f^{+}(u)-g^{+}(v)\right]}}{\cosh ^{2} \beta t} f^{+^{\prime}}(u) g^{+^{\prime}}(v), & r \geq r_{0},\end{cases}
$$

which diverges like $\left(\tau_{0}-\tau\right)^{-1}$, as $r \rightarrow r_{0}$, as long as $f^{ \pm^{\prime}}(u) g^{ \pm^{\prime}}(v) \neq 0$, as one can show from Eqs.(A.9) and (A.10). When only the outgoing null dust fluid exists, the singularity degenerates to a non-scalar curvature singularity, as it can be shown that now all the fourteen scalars made out of the Riemann tensor are zero. However, in any case the singularity is strong in the sense that the distortion, which is equal to the twice integral of the tetrad components of the Riemann tensor, becomes unbounded, for example,

$$
\begin{aligned}
\iint R_{(0)(2)(0)(2)}^{ \pm} d \tau d \tau= & \pm \frac{\sqrt{2} \beta}{4} \iint \frac{1}{\cosh \beta t} \\
& \times\left[D_{+}(A) e^{ \pm \beta(t-2 R)} f^{ \pm^{\prime}}(u)\right. \\
& \left.-D_{-}(A) e^{\mp \beta(t+2 R)} g^{ \pm^{\prime}}(v)\right] d \tau d \tau \\
\sim & g^{ \pm^{\prime}}(v) \ln \left(\tau_{0}-\tau\right)
\end{aligned}
$$

as $r \rightarrow r_{0}$. It is interesting to note that, although the tetrad components of $T_{\mu \nu}$ are singular as $r \rightarrow r_{0}$, the scalar $T^{\mu \nu} T_{\mu \nu}$ does not. In fact, from Eq. 4.13) it can be shown that

$$
\begin{aligned}
T_{\mu \nu}^{ \pm} T^{ \pm \mu \nu} & =T_{(a)(b)}^{ \pm} T^{ \pm(a)(b)}=2 \rho_{ \pm}^{\text {in }} \rho_{ \pm}^{\text {out }}= \\
& =-\frac{\beta^{2} f^{ \pm^{\prime}}(u) g^{ \pm^{\prime}}(v)}{2 \cosh ^{2} \beta t} \sim\left(\tau_{0}-\tau\right)
\end{aligned}
$$

as $r \rightarrow r_{0}$. Thus, the formation of the spacetime singularity is mainly due 
to the focus of the corresponding gravitational fields. This is different from what we can get from Eq.(3.9) for the test particles.

$$
\text { B. } b(t)=+\beta t
$$

When $b(t)=+\beta t$, from Eqs.(4.2) - (4.5) we find that

$$
\begin{aligned}
\Omega & =f^{-}(u)+g^{-}(v)+2 \beta R, \\
h & =-\beta(t-R)-\ln C_{0}, \\
\Phi & =\beta(t-R)-\ln C_{0},
\end{aligned}
$$

for $r \leq r_{0}$, and

$$
\begin{aligned}
\Omega & =f^{+}(u)+g^{+}(v)-2 \beta R, \\
h & =-\beta(t+R)-\ln C_{0}, \\
\Phi & =\beta(t+R)-\ln C_{0},
\end{aligned}
$$

for $r \geq r_{0}$. Substituting Eqs.(4.18) and (4.19) into Eq.(4.7), we find that

$$
\begin{aligned}
& \rho_{-}^{\text {out }}=-\sqrt{2} \beta g^{-^{\prime}}(v), \quad \rho_{-}^{\text {in }}=0,\left(r \leq r_{0}\right) \\
& \rho_{+}^{\text {out }}=0, \quad \rho_{+}^{\text {in }}=-\sqrt{2} \beta{f^{+}}^{\prime}(u),\left(r \geq r_{0}\right) .
\end{aligned}
$$

The above expressions show that in the region $r \leq r_{0}$ now there exists only outgoing dust cloud, while in the region $r \geq r_{0}$ only ingoing. With the same arguments as those given in the last subsection, we take the tetrad frames 
given by Eqs.(A.6) and (A.7) as a good approximation to the corresponding PPON of Eq.(4.6), when $f^{ \pm}(u), g^{ \pm}(v)$, and their first derivatives are very small. Then, projecting the EMT onto this frame, we find that the nonvanishing tetrad components of it are given by

$$
\begin{aligned}
& T_{(0)(0)}^{-}=T_{(1)(1)}^{-}=-T_{(0)(1)}^{-}=-\frac{\beta}{\sqrt{2}} D_{-}(A) g^{-\prime}(v),\left(r \leq r_{0}\right), \\
& T_{(0)(0)}^{+}=T_{(1)(1)}^{+}=T_{(0)(1)}^{+}=-\frac{\beta}{\sqrt{2}} D_{+}(A) f^{+^{\prime}}(u),\left(r \geq r_{0}\right),
\end{aligned}
$$

with $A$ being given by Eqs.(2.6) and (2.13), and $D_{ \pm}$are defined by Eq.(4.14). Clearly, as $r \rightarrow r_{0}$, these components all diverge, although at the initial $t=-\infty, r \neq r_{0}$ they are finite. That is, the perturbations, similar to the last subcase, turn the CEHs into spacetime curvature singularities. The nature of the singularity is a non-scalar one, as one can show that now all the fourteen scalars built from the Riemann tensor are zero. However, the singularity is strong in the sense that the distortion diverges like $\ln \left(\tau_{0}-\tau\right)$ as $r \rightarrow r_{0}$, as we can see from the following integrations,

$$
\begin{aligned}
\iint R_{(0)(2)(0)(2)}^{-} d \tau d \tau & =\frac{1}{\sqrt{2} \beta} \iint \frac{g^{-\prime}(v)}{\left(r_{0}-r\right)^{2}} D_{-}(A) d \tau d \tau \\
& \sim \ln \left(\tau_{0}-\tau\right) \\
\iint R_{(0)(2)(0)(2)}^{+} d \tau d \tau & =\frac{1}{\sqrt{2} \beta} \iint \frac{f^{+^{\prime}}(u)}{\left(r-r_{0}\right)^{2}} D_{+}(A) d \tau d \tau \\
& \sim \ln \left(\tau_{0}-\tau\right) .
\end{aligned}
$$




$$
\text { C. } b(t)=-\beta t
$$

When $b(t)=-\beta t$, Eqs.(4.2) - (4.5) yield

$$
\begin{aligned}
\Omega & =f^{-}(u)+g^{-}(v)+2 \beta R, \\
h & =\beta(t+R)-\ln C_{0}, \\
\Phi & =-\beta(t+R)-\ln C_{0},
\end{aligned}
$$

for $r \leq r_{0}$, and

$$
\begin{aligned}
\Omega & =f^{+}(u)+g^{+}(v)-2 \beta R, \\
h & =\beta(t-R)-\ln C_{0}, \\
\Phi & =-\beta(t-R)-\ln C_{0},
\end{aligned}
$$

for $r \geq r_{0}$. Substituting Eqs.(4.23) and (4.24) into Eq.(4.7), we find

$$
\begin{aligned}
& \rho_{-}^{\text {out }}=0, \quad \rho_{-}^{\text {in }}=\sqrt{2} \beta f^{-^{\prime}}(u),\left(r \leq r_{0}\right), \\
& \rho_{+}^{\text {out }}=\sqrt{2} \beta g^{+^{\prime}}(v), \quad \rho_{+}^{\text {in }}=0,\left(r \geq r_{0}\right) .
\end{aligned}
$$

Thus, in the present case in the region $r \leq r_{0}$ there exists only ingoing dust cloud, while in the region $r \geq r_{0}$ only outgoing. Therefore, now the dust clouds cannot be considered as perturbations, but rather than as emission of null fluids from the CEHs. To study the stability of the CEHs in this case we have to consider other kinds of perturbations. However, the following considerations indicate that they may be not stable, too. Projecting the 
EMT onto the frame given by Eqs.(A.6) and (A.7), we find that

$$
\begin{aligned}
& T_{(0)(0)}^{-}=T_{(1)(1)}^{-}=T_{(0)(1)}^{-}=\frac{\beta}{\sqrt{2}} D_{+}(A) f^{-^{\prime}}(u),\left(r \leq r_{0}\right), \\
& T_{(0)(0)}^{+}=T_{(1)(1)}^{+}=-T_{(0)(1)}^{+}=\frac{\beta}{\sqrt{2}} D_{+}(A) g^{+^{\prime}}(v),\left(r \geq r_{0}\right) .
\end{aligned}
$$

From Eq.(4.14) and the above expressions we can see that the back reaction of the emission also turns the CEHs into spacetime singularities. Similar to the last subcase, the nature of the singularity is a non-scalar one but strong, as the distortion also diverges as $r \rightarrow r_{0}$,

$$
\begin{aligned}
\iint R_{(0)(2)(0)(2)}^{-} d \tau d \tau & =-\frac{1}{\sqrt{2} \beta} \iint \frac{f^{-\prime}(u)}{\left(r_{0}-r\right)^{2}} D_{+}(A) d \tau d \tau \\
& \sim \ln \left(\tau_{0}-\tau\right) \\
\iint R_{(0)(2)(0)(2)}^{+} d \tau d \tau & =-\frac{1}{\sqrt{2} \beta} \iint \frac{g^{+^{\prime}}(v)}{\left(r-r_{0}\right)^{2}} D_{-}(A) d \tau d \tau \\
& \sim \ln \left(\tau_{0}-\tau\right) .
\end{aligned}
$$

Thus, for the perturbations that have non-vanishing components along the ingoing null geodesics defined by $l_{\mu}$ in the region $r \leq r_{0}$, or for the perturbations that have non-vanishing components along the outgoing null geodesics defined by $n_{\mu}$ in the region $r \geq r_{0}$, we would expect that the CEHs will be turned into spacetime singularities.

\section{CONCLUDING REMARKS}

In this paper, we have considered the stability of the CEHs for a class of non-static global cosmic strings found recently by Gregory 9, and found 
that they are not stable against perturbations. In particular, the back reaction of null dust fluids will turn them into spacetime singularities. Thus resulted singularities are strong in the sense that the distortion of test particles diverges when these singular hypersurfaces are approaching.

Recently, we have shown that the CEHs of topological domain walls are also not stable against massless scalar field [16] and null dust fluids [17]. Thus, a natural question is that: Are all the cosmological event horizons not stable? If some are but others not, what are the criteria for them? It was exactly this consideration that motivated us to study the test particles in Sec. III, at the aim of generalizing the HK conjecture to the study of the stability of the CEHs. Comparing the results obtained in Sec. III with the ones obtained in Sec. IV for real perturbations, we can see that the HK conjecture can be used directly to the study of the stability of CEHs (as far as the examples considered in this paper are concerned), except for the case where $b(t)=\ln [\cosh (\beta t)]$. In the latter case, although the study of the test particles gives a correct prediction for the nature of the resulted singularities, but the quantity $T_{\mu \nu} T^{\mu \nu}$ for test particle diverges, while for the real perturbations it does not. As a matter of fact, the divergence of the Kretschmann scalar is mainly due to the non-linear interaction of gravitational fields for the real perturbations. Clearly, to properly form the conjecture, more examples need to be considered. 
Finally, we would like to note that although the CEHs found by Gregory are not stable, and after the back reaction of perturbations is taken into account, they will be turned into spacetime singularities, the hope that the time-dependence of the spacetimes for global cosmic strings may remove the singular nature of the corresponding static ones has not been shown completely negative. As we mentioned in the introduction, the class of spacetimes considered by Gregory is not the most general spacetimes for non-static global cosmic strings. In some cases, one may expect that the gravitational and particle radiation is so strong that the gravitational field of a global string may be well dilated before any spacetime singularity or event horizon is formed. Spacetimes with cylindrical symmetry are quite different from those with spherical symmetry. As a matter of fact, in the former case gravitational radiation generally always exists.

\section{APPENDIX}

In this appendix, we shall briefly review some main properties of the spacetimes given by

$$
d s^{2}=e^{2 A(r)} d t^{2}-d r^{2}-e^{2[A(r)+b(t)]} d z^{2}-C^{2}(r) d \theta^{2},
$$

where $A, b$ and $C$ are arbitrary functions of their indicated arguments. 
The corresponding Lagrangian of the radial geodesics is given by

$$
\mathcal{L}=\frac{1}{2}\left(\frac{d s}{d \tau}\right)^{2}=\frac{1}{2}\left[e^{2 A(r)} \dot{t}^{2}-\dot{r}^{2}\right]
$$

where an overdot denotes the ordinary derivative with respect to the parameter $\tau$ of the geodesics. For time-like geodesics, $\tau$ can be identified as the proper time of the test particles. Since $\mathcal{L}$ is independent of $t$, the quantity $E$ given by

$$
E \equiv \frac{\partial \mathcal{L}}{\partial \dot{t}}=e^{2 A(r)} \dot{t}
$$

is an integration constant, which represents the total energy of the test particles for time-like geodesics. Substituting Eq.(A.3) into Eq.(A.2), we find

$$
\dot{r}=\epsilon\left(\frac{E^{2}}{e^{2 A(r)}}-2 \mathcal{L}\right)^{1 / 2},(\epsilon= \pm 1),
$$

where $\epsilon=+1$ corresponds to the out-going geodesics, while $\epsilon=-1$ to the ingoing geodesics. By properly choosing the parameter $\tau$, we can always make $2 \mathcal{L}=+1,0,-1$, respectively, for time-like, null, and space-like geodesics. In the following, we shall consider only time-like geodesics. Then, the motion of the test particles has the first integral

$$
\dot{t}=\frac{E}{e^{2 A(r)}}, \quad \dot{r}=\epsilon\left(\frac{E^{2}}{e^{2 A(r)}}-1\right)^{1 / 2} .
$$

Denoting the tangent vector to the geodesics by $\lambda_{(0)}^{\mu}$,

$$
\lambda_{(0)}^{\mu} \equiv \frac{d x^{\mu}}{d \tau}=\dot{t} \delta_{t}^{\mu}+\dot{r} \delta_{r}^{\mu}=\dot{t} \delta_{t}^{\mu}+e^{-a(R)} \dot{r} \delta_{R}^{\mu},
$$


we can construct other three orthogonal space-like vectors

$$
\begin{aligned}
& \lambda_{(1)}^{\mu}=e^{-A(r)} \dot{r} \delta_{t}^{\mu}+e^{A(r)} \dot{t} \delta_{r}^{\mu}=e^{-a(R)} \dot{r} \delta_{t}^{\mu}+\dot{t} \delta_{R}^{\mu}, \\
& \lambda_{(2)}^{\mu}=e^{-[A(r)+b(t)]} \delta_{z}^{\mu}, \quad \lambda_{(3)}^{\mu}=C^{-1}(r) \delta_{\theta}^{\mu} .
\end{aligned}
$$

Then, it can be shown that

$$
\lambda_{(i)}^{\mu} \lambda_{(j) \mu}=\eta_{i j}, \quad \lambda_{(i) ; \nu}^{\mu} \lambda_{(0)}^{\nu}=0,(i, j=0,1,2,3),
$$

where $\eta_{i j}$ is the Minkowski metric. The above equations show that the four unit vector $\lambda_{(i)}^{\mu}$ form a freely-falling frame or parallel-propagated orthogonal frame (PPON) along the time-like geodesics.

For the particular solutions of $A(r)$ given by Eqs.(2.6) and (2.13), Eq.(A.5) has the following integration,

$$
\begin{aligned}
e^{-2 \beta R} & =\beta^{2}\left(r_{0}-r\right)^{2}=\beta^{2}\left(\tau_{0}^{2}-\tau^{2}\right), \\
e^{2 \beta t} & =\frac{\tau_{0}+\tau}{\tau_{0}-\tau},\left(r \leq r_{0}\right),
\end{aligned}
$$

for $r \leq r_{0}$, and

$$
\begin{aligned}
e^{2 \beta R} & =\beta^{2}\left(r-r_{0}\right)^{2}=\beta^{2}\left(\tau_{0}^{2}-\tau^{2}\right), \\
e^{2 \beta t} & =\frac{\tau_{0}+\tau}{\tau_{0}-\tau},\left(r \geq r_{0}\right),
\end{aligned}
$$

for $r \geq r_{0}$, where $\tau_{0}$ is chosen such that when $r \rightarrow r_{0}$, we have $\tau \rightarrow \tau_{0}$.

On the other hand, the corresponding Kretschmann scalar to the metric 
(A.1) is given by

$$
\begin{aligned}
\mathcal{R} \equiv & R_{\alpha \beta \gamma \lambda} R^{\alpha \beta \gamma \lambda}=4\left\{\left(\frac{C^{\prime \prime}}{C}\right)^{2}+2\left(\frac{A^{\prime} C^{\prime}}{C}\right)^{2}\right\} \\
& +4\left\{2\left(A^{\prime \prime}+A^{\prime 2}\right)^{2}+\left[A^{\prime 2}-\left(b^{\prime \prime}+b^{\prime 2}\right) e^{-2 A}\right]^{2}\right\} .
\end{aligned}
$$

Choosing a null tetrad frame, on the other hand, as

$$
\begin{aligned}
l_{\mu} & =\frac{1}{\sqrt{2}}\left(e^{A(r)} \delta_{\mu}^{t}+\delta_{\mu}^{r}\right)=\frac{e^{a(R)}}{\sqrt{2}}\left(\delta_{\mu}^{t}+\delta_{\mu}^{R}\right), \\
n_{\mu} & =\frac{1}{\sqrt{2}}\left(e^{A(r)} \delta_{\mu}^{t}-\delta_{\mu}^{r}\right)=\frac{e^{a(R)}}{\sqrt{2}}\left(\delta_{\mu}^{t}-\delta_{\mu}^{R}\right), \\
m_{\mu} & =\frac{1}{\sqrt{2}}\left[e^{A(r)+b(t)} \delta_{\mu}^{z}+i C(r) \delta_{\mu}^{\theta}\right], \\
\bar{m}_{\mu} & =\frac{1}{\sqrt{2}}\left[e^{A(r)+b(t)} \delta_{\mu}^{z}-i C(r) \delta_{\mu}^{\theta}\right],
\end{aligned}
$$

we find that the non-vanishing Weyl scalars are given by

$$
\begin{aligned}
\Psi_{2} & =-\frac{1}{2} C_{\mu \nu \lambda \delta}\left[l^{\mu} n^{\nu} l^{\lambda} n^{\delta}-l^{\mu} n^{\nu} m^{\lambda} \bar{m}^{\delta}\right] \\
& =\frac{1}{12}\left\{-\frac{C^{\prime \prime}}{C}+A^{\prime \prime}+\frac{C^{\prime}}{C} A^{\prime}+\left(b^{\prime \prime}+b^{2}\right) e^{-2 A}\right\}, \\
\Psi_{0} & =-C_{\mu \nu \lambda \delta} l^{\mu} m^{\nu} l^{\lambda} m^{\delta}=-3 \Psi_{2}, \\
\Psi_{4} & =-C_{\mu \nu \lambda \delta} n^{\mu} \bar{m}^{\nu} n^{\lambda} \bar{m}^{\delta}=-3 \Psi_{2} .
\end{aligned}
$$

Thus, we have

$$
\Psi_{0} \Psi_{4}=9 \Psi_{2}^{2} .
$$

Then, according to the theorem given in [18, we find that the metric (A.1) is always Petrov type $\mathrm{D}$, except for the degenerate case where $\Psi_{2}=0$, which is Petrov type $\mathrm{O}$. 
Finally, we would like to note that $l_{\mu}\left(n_{\mu}\right)$ defines an ingoing (outgoing) radial null geodesic congruence [15].

\section{Acknowledgment}

The financial assistance from CNPq (AW), and the ones from CLAF-CNPq (JACN) and UMSA (JACN), are gratefully acknowledged.

\section{References}

[1] A. Vilenkin and E.P.S. Shellard, Cosmic Strings and other Topological Defects, (Cambridge University Press, Cambridge, 1994); M.B. Hindmarsh and T.W.B. Kibble, Rep. Prog. Phys. 58, 477 (1995).

[2] T.W.B Kibble, J. Phys. A9, 1387 (1976).

[3] R. Gregory, Phys. Rev. Lett. 59, 740 (1987).

[4] D. Harari and P. Sikivie, Phys. Rev. D37, 3438 (1988); A.G. Cohen and D.B. Kaplan, Phys. Lett. B215, 67 (1988); R. Gregory, ibid. 215, 663 (1988); D. Harari and A.P. Polychronakos, ibid., 240, 55 (1989); G.W. Gibbons, M.E. Ortiz, and F.R. Ruiz, Phys. Rev. D39, 1546 (1989).

[5] D.P. Bennett and F.R. Bouchet, Phys. Rev. D43, 2733 (1991); R.R. Caldwell and B. Allen, ibid. 45, 1169 (1992). 
[6] A.Z. Wang and N.O. Santos, Class. Quantum Grav. 13, 715 (1996).

[7] R.A. Battye and E.P.S. Shellard, Phys. Rev. D53, 1811 (1996).

[8] A. Banerjee, N. Banerjee, and A.A. Sen, Phys. Rev. D53, 5508 (1996).

[9] R. Gregory, Phys. Rev. D54, 4955 (1996).

[10] G.W. Gibbons and S.W. Hawking, Phys. Rev. D15, 2738 (1977).

[11] D. Kramer, H. Stephani, E. Herlt, and M. MacCallum, Exact Solutions of Einstein's Field Equations, edited by E. Schmutzer ( Cambridge University Press, Cambridge, England, 1980).

[12] S.W. Hawking and G.F.R. Ellis, The Large Scale Structure of Spacetime, (Cambridge University Press, Cambridge, 1973).

[13] T.M. Helliwell and D.A. Konkowski, Phys. Rev. D46, 1424 (1992), and references therein.

[14] D.A. Konkowski and T.M. Helliwell, Phys. Rev. D54, 7898 (1996), and references therein.

[15] P.S. Letelier and A.Z. Wang, Phys. Rev. D49, 5105 (1994).

[16] A.Z. Wang, Phys. Lett. B277, 49 (1992); Phys. Rev. D48, 2591 (1993).

[17] A.Z. Wang and P.S. Letelier, Phys. Rev. D52, 1800 (1995). 
[18] S. Chandrasekhar and B.C. Xanthopoulos, Proc. Roy. Soc. Lond. A408, 175 (1986). 